\title{
Maternal nutrient restriction in guinea pigs leads to fetal growth restriction with evidence for chronic hypoxia
}

\author{
Alexander A. Elias ${ }^{1,2}$, Yohei Maki ${ }^{1}$, Brad Matushewski ${ }^{1}$, Karen Nygard ${ }^{4}$, Timothy R.H. Regnault ${ }^{1,2,5}$ and \\ Bryan S. Richardson ${ }^{1,2,3,5}$
}

BACKGROUND: We determined whether maternal nutrient restriction (MNR) in guinea pigs leading to fetal growth restriction (FGR) impacts markers for tissue hypoxia, implicating a mechanistic role for chronic hypoxia.

METHODS: Guinea pigs were fed ad libitum (Control) or 70\% of the control diet before pregnancy, switching to $90 \%$ at mid-pregnancy (MNR). Near term, hypoxyprobe-1 (HP-1), a marker of tissue hypoxia, was injected into pregnant sows. Fetuses were then necropsied and liver, kidney, and placental tissues were processed for erythropoietin (EPO), EPO-receptor (EPOR), and vascular endothelial growth factor (VEGF) protein levels, and for HP-1 immunoreactivity (IR).

RESULTS: FGR-MNR fetuses were 36\% smaller with asymmetrical growth restriction compared to controls. EPO and VEGF protein levels were increased in the female FGR-MNR fetuses, providing support for hypoxic stimulus and linkage to increased erythropoiesis, but not in the male FGR-MNR fetuses, possibly reflecting a weaker link between oxygenation and erythropoiesis. HP-1 IR was increased in the liver and kidneys of both male and female FGR-MNR fetuses as an index of local tissue hypoxia, but with no changes in the placenta.

CONCLUSION: Chronic hypoxia is likely to be an important signaling mechanism for the decreased fetal growth seen with maternal undernutrition and appears to be postplacental in nature.

$\mathbf{F}$ etal growth is dependent on placental development and maternal nutrition, which impacts substrate availability, with placental insufficiency and maternal undernutrition being primary causes of fetal growth restriction (FGR) $(1,2)$. Clinical studies of placental insufficiency-related FGR demonstrate aberrant placental vascularization and decreases in umbilical blood flow $(3,4)$, leading to chronic fetal hypoxemia as a primary signaling mechanism for growth restriction in these pregnancies $(5,6)$. This has led to animal models of placental insufficiency induced by restricting placental blood flow and leading to FGR with variable hypoxemia and nutrient restriction $(7,8)$. Clinical studies in populations subjected to food restriction (9) and in mothers deemed underweight $(10,11)$ support the notion that maternal undernutrition is also causative for FGR dependent upon severity and timing before conception and through pregnancy. This has led to animal models of maternal nutrient restriction (MNR) with induced FGR that show aberrant placental development with reduced surface area for nutrient exchange, increased barrier thickness, and altered vascularization $(12,13)$. These structural alterations are also seen in human FGR (14) and have been associated with reduced transport for glucose, amino acids, and lipids as nutritional cues for related FGR $(13,15)$. However, whether fetal oxygenation is also decreased as a signaling mechanism for growth restriction with these MNR-related placental alterations has yet to be determined.

Guinea pigs deliver precocial young after a relatively long pregnancy, with many developmental events occurring during fetal life similar to those in humans (16). Moderate MNR in guinea pigs at $70 \%$ of an ad libitum diet from 4 weeks before conception until mid-pregnancy increasing to $90 \%$ thereafter has therefore been studied for modeling human FGR $(12,17,18)$. In these studies fetal weights have been shown to be decreased by as much as $40 \%$ near term with asymmetric growth restriction, polycythemia, and hypoglycemia $(12,17,18)$, which are also well associated with FGR in humans $(2,5)$. These findings along with the structural changes and altered vascular development in the placentas of these animals $(12,13)$ are suggestive of the occurrence of chronic hypoxemia, although more compelling evidence is lacking.

In the present study, we have determined whether moderate MNR in guinea pigs as a causative factor for FGR also impacts markers for tissue hypoxia to test the hypothesis that these markers will be increased, further implicating chronic hypoxia as a primary signaling mechanism here. Protein levels for erythropoietin (EPO) and its receptor (EPOR) have been assessed, since these are upregulated to promote erythropoiesis in response to systemic hypoxia, and for VEGF, since this

\footnotetext{
${ }^{1}$ Department of Obstetrics and Gynecology, University of Western Ontario, London, Ontario, Canada; ${ }^{2}$ Department of Physiology and Pharmacology, University of Western Ontario, London, Ontario, Canada; ${ }^{3}$ Department of Pediatrics, University of Western Ontario, London, Ontario, Canada; ${ }^{4}$ Biotron Experimental Climate Change Research Centre, University of Western Ontario, London, Ontario, Canada; ${ }^{5}$ Children's Health Research Institute, Schulich School of Medicine and Dentistry, University of Western Ontario, London, Ontario, Canada. Correspondence: B.S. Richardson (brichar1@uwo.ca)

Received 5 October 2016; accepted 6 March 2017; advance online publication 24 May 2017. doi:10.1038/pr.2017.92
} 


\section{Articles | Elias et al.}

Table 1. Fetal population characteristics

\begin{tabular}{|c|c|c|c|c|c|c|c|}
\hline & \multicolumn{2}{|c|}{ Male } & \multicolumn{2}{|c|}{ Female } & \multicolumn{3}{|c|}{$P($ ANOVA $)$} \\
\hline & AGA-Control & FGR-MNR & AGA-Control & FGR-MNR & G & $S$ & $\mathrm{G} \times \mathrm{S}$ \\
\hline Fetal wt (g) & $101 \pm 3$ & $64 \pm 4$ & $101 \pm 3$ & $65 \pm 4$ & $* * *$ & NS & NS \\
\hline Brain wt (g) & $2.76 \pm 0.07$ & $2.45 \pm 0.08$ & $2.72 \pm 0.08$ & $2.36 \pm 0.07$ & $* *$ & NS & NS \\
\hline Liver wt (g) & $4.6 \pm 0.2$ & $2.7 \pm 0.3$ & $4.7 \pm 0.4$ & $2.8 \pm 0.2$ & $* * *$ & NS & NS \\
\hline Brain/liver wt & $0.60 \pm 0.03$ & $0.98 \pm 0.07$ & $0.62 \pm 0.05$ & $0.88 \pm 0.05$ & $* * *$ & NS & NS \\
\hline Placental wt (g) & $7.3 \pm 0.5$ & $5.4 \pm 0.5$ & $6.7 \pm 0.4$ & $5.2 \pm 0.4$ & $* *$ & NS & NS \\
\hline Placental/fetal wt & $0.073 \pm 0.006$ & $0.088 \pm 0.009$ & $0.067 \pm 0.005$ & $0.082 \pm 0.006$ & * & NS & NS \\
\hline
\end{tabular}

AGA, appropriate for gestational age; FGR, fetal growth restriction; G, group; MNR, maternal nutrient restriction; NS, not significant; S, sex.

Data presented as means \pm SEM; ${ }^{*} P<0.05,{ }^{* *} P<0.01,{ }^{* *} P<0.001 ; n$ values were 8 male and 8 female AGA-Control and 8 male and 8 female FGR-MNR fetuses.

is upregulated to promote angiogenesis in response to local tissue hypoxia (19). Immunoreactivity for Hypoxyprobe-1 (HP-1) has additionally been assessed as a widely used marker of tissue hypoxia (20-22).

\section{RESULTS}

\section{Fetal Population Characteristics}

While 12 guinea pig sows were bred under ad libitum feeding conditions and 18 under MNR feeding conditions, 3 animals from each of these groups failed to become pregnant, and 3 MNR animals delivered preterm. The remaining 9 control and $12 \mathrm{MNR}$ animals had pregnancies continuing to necropsy at 60/61 days of gestation with 31 and 42 fetuses, respectively, whose growth characteristics have been reported previously (18). As noted, we used $80 \mathrm{~g}$ as our threshold for categorizing appropriate for gestational age (AGA) and FGR fetal weights, resulting in 20 AGA-Control fetuses (10 males and 10 females) and 25 FGR-MNR fetuses (11 males and 14 females), with the growth characteristics from these animals also previously reported (18). The 16 AGA-Control fetuses and 16 FGR-MNR fetuses presently studied were representative of the mean fetal weights from these select cohort groups, with their population characteristics shown in Table 1. Changes in these population characteristics between FGR-MNR fetuses and AGA-Control fetuses were similar for both males and females, with no sexrelated differences noted. Briefly, FGR-MNR fetal weights were decreased by $\sim 36 \%$ compared to those of the AGA-Control fetuses. FGR-MNR brain weights were decreased, but less so, by $\sim 12 \%$, while FGR-MNR liver weights were markedly decreased by $\sim 40 \%$. Accordingly, the brain/liver weight ratio as a measure of asymmetrical growth was increased by $~ 50 \%$ in the FGR-MNR fetuses compared to those in the AGA-Control fetuses. Placental weights were decreased in the FGR-MNR fetuses by $\sim 24 \%$, but less so than the fetal weights, with the placental/fetal weight ratios thus increased by $\sim 20 \%$.

\section{Fetal EPO, EPOR, and VEGF Protein Levels}

EPO showed both group and sex-related differences, whereby levels in the FGR-MNR females were increased in the liver by $\sim 1.9$-fold, in the kidney by $\sim 2.5$-fold, and in the placenta by $\sim 1.7$-fold when compared to that of the AGA-Control females (Figure 1). However, there were no significant changes in EPO
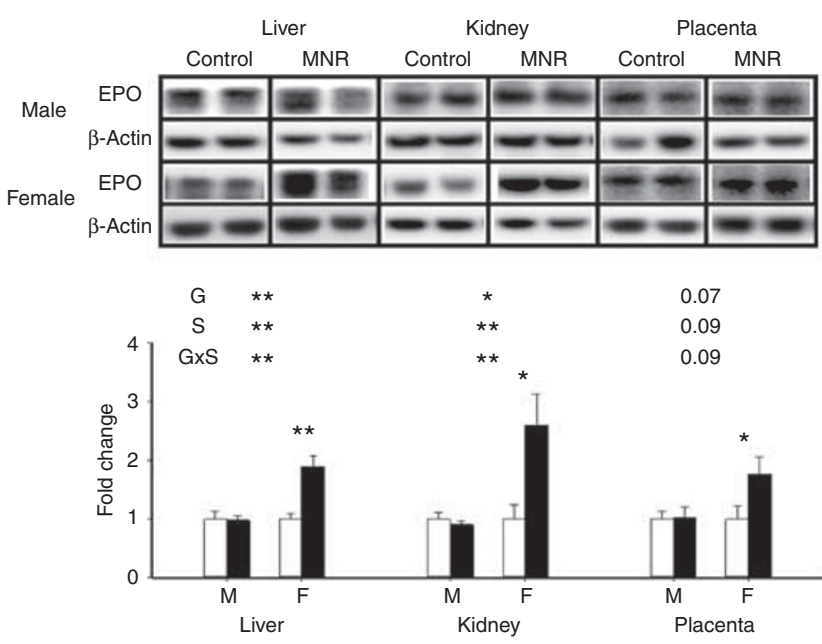

Figure 1. EPO protein expression in fetal liver, kidney, and placenta. Representative immunoblots from male and female AGA-Control and FGR-MNR animals are displayed. Density of EPO normalized to $\beta$-actin and presented as the mean fold change \pm SEM in male and female FGR-MNR fetuses (gray bars, $n=8$ and 8 ) compared with male and female AGA-Control fetuses (open bars, $n=8$ and 8). ${ }^{*} P<0.05$, ${ }^{*} P<0.01$; AGA, appropriate for gestational age; EPO, erythropoietin; FGR, fetal growth restriction; G, group; MNR, maternal nutrient restriction; NS, not significant; $S$, sex.

levels between FGR-MNR males and AGA-Control males for any of the tissues studied (Figure 1). EPOR was not significantly changed by either group or sex for any of the tissues studied, although levels in the liver were increased in the FGR-MNR females by $\sim 1.6$-fold, while the levels decreased in the FGR-MNR males by $\sim 50 \%$ from respective AGA-Control levels (Figure 2). VEGF also showed both group and sex-related differences, whereby levels in the FGRMNR females were increased in the liver by approximately twofold, while the levels in the FGR-MNR males were increased in the placenta by approximately twofold from respective AGA-Control levels, but with no other significant changes (Figure 3).

\section{Hypoxyprobe-1 Immunoreactivity}

Representative images for HP-1 IR are shown in Figure 4 and illustrate the presence of positive staining in both the FGR- 


\section{Maternal undernutrition in guinea pigs $\quad$ Articles}
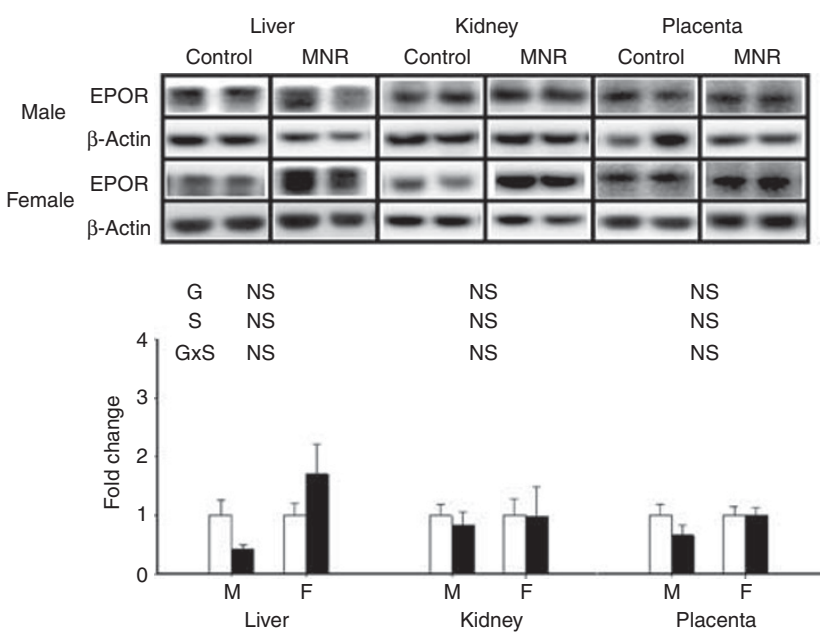

Figure 2. EPOR protein expression in fetal liver, kidney, and placenta. Representative immunoblots from male and female AGA-Control and FGR-MNR animals are displayed. Density of EPOR normalized to $\beta$-actin and presented as the mean fold change \pm SEM in male and female FGR-MNR fetuses (gray bars, $n=8$ and 8) compared with male and female AGA-Control fetuses (open bars, $n=8$ and 8). AGA, appropriate for gestational age; EPOR, erythropoietin receptor; FGR, fetal growth restriction; G, group; MNR, maternal nutrient restriction; NS, not significant; $\mathrm{S}$, sex.

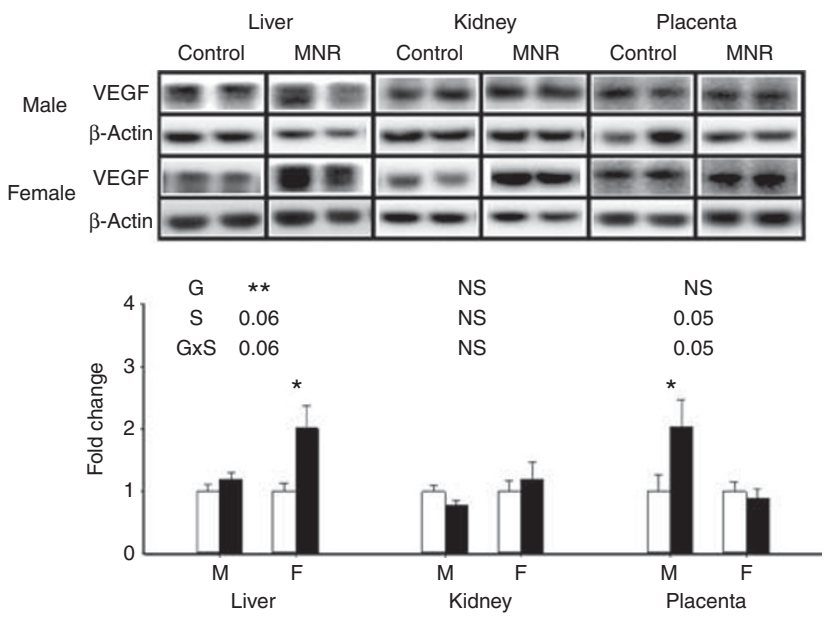

Figure 3. VEGF protein expression in fetal liver, kidney and placenta. Representative immunoblots from male and female AGA-Control and FGR-MNR animals are displayed. Density of VEGF normalized to $\beta$-actin and presented as the mean fold change \pm SEM in the male and female FGR-MNR fetuses (gray bars, $n=8$ and 8) compared with male and female AGA-Control fetuses (open bars, $n=8$ and 8 ). ${ }^{*} P<0.05$, ${ }^{* *} P<0.01$; AGA, appropriate for gestational age; FGR, fetal growth restriction; G, group; MNR, maternal nutrient restriction; NS, not significant; S, sex; VEGF, vascular endothelial growth factor.

MNR and AGA-Control animals for each of the liver, kidney, and placenta, indicating some level of local tissue hypoxia even under normal physiologic conditions in the fetus. Tissue levels of HP-1 shown in Figure 5 were similar for the male and female AGA-Control fetuses in respective tissues and tissue regions, while changes in these levels in the FGR-MNR fetuses were similar for both males and females, with no sex- related differences noted. In the liver, HP-1 staining was widely seen within the hepatocytes, but tissue levels of HP-1 as an index of local tissue hypoxia were increased in the FGR-MNR fetuses by approximately fourfold compared to that in AGA-Control fetuses. In the kidney, HP-1 was widely seen within the cortex, but much more in the proximal convoluted tubules than in the glomeruli by $\sim 80$ fold on average for the AGA-Controls $(P<0.01$ analyzed using paired $t$-test). Tissue levels of HP-1 were increased in the FGR-MNR fetuses by $\sim 15$-fold in the glomeruli and by $\sim 4$-fold in the proximal convoluted tubules compared to respective AGA-Control fetuses. In the placenta, image analysis was confined to the lobulated labyrinthine region since this is the largest structural region near term and is where nutrient and gas exchange primarily occurs (23). HP-1 was again widely seen, but more so peripherally than centrally within the labyrinthine lobules by approximately fivefold on average for the AGA-Controls $(P<0.05$ analyzed using paired $t$-test). However, in contrast to the liver and kidney there was no increase in tissue levels of HP-1 in the placentas of the FGR-MNR fetuses as studied.

\section{DISCUSSION}

As we (18) and others (12) have reported, moderate MNR in guinea pigs results in a decrease in fetal weights by 30 to $40 \%$ on average for all MNR pregnancies necropsied near term. We set a threshold of $\geq 80 \mathrm{~g}$ or $<80 \mathrm{~g}$ for categorizing AGAControl and FGR-MNR fetal cohorts, respectively, which was close to the 10th percentile for the population weight distribution of the Control fetuses (18). This then better reflects the human situation where AGA and small-forgestational-age (SGA) birth weight distributions are separate and often delineated by the 10th percentile adjusted for gestational age $(2,6)$. While it is recognized that some SGA infants will be constitutionally small rather than growth restricted, this classification is widely used for denoting increased risk for FGR and adverse outcomes.

EPO binds to its cognate receptor on erythroid progenitor cells, stimulating their survival and differentiation, with the rate of red blood cell production thereby determined by blood/tissue EPO levels $(19,24)$. EPO synthesis is inversely related to oxygen availability in tissues, with the main determinant being the transcriptional activity of its gene by hypoxia-inducible factor 1 (HIF-1) (19,24). During fetal development, EPO synthesis gradually changes from hepatocytes stimulating erythropoiesis in the liver to renal EPOproducing cells in the cortex and outer medulla stimulating erythropoiesis in the bone marrow, with the timing for this transition being species dependent (25-27). However, secretion of small amounts of EPO has also been identified in other fetal tissues, and for the placenta this can become substantial in response to fetal hypoxemia $(25,26)$. In the present study, the FGR-MNR female fetuses exhibited increased EPO levels in the liver, kidney, and placenta in keeping with a degree of local hypoxia in these tissues and supporting our conjecture of relative fetal hypoxemia in FGR-MNR fetuses. However, EPO levels in the male FGR-MNR fetuses were little changed, 


\section{Articles | Elias et al.}

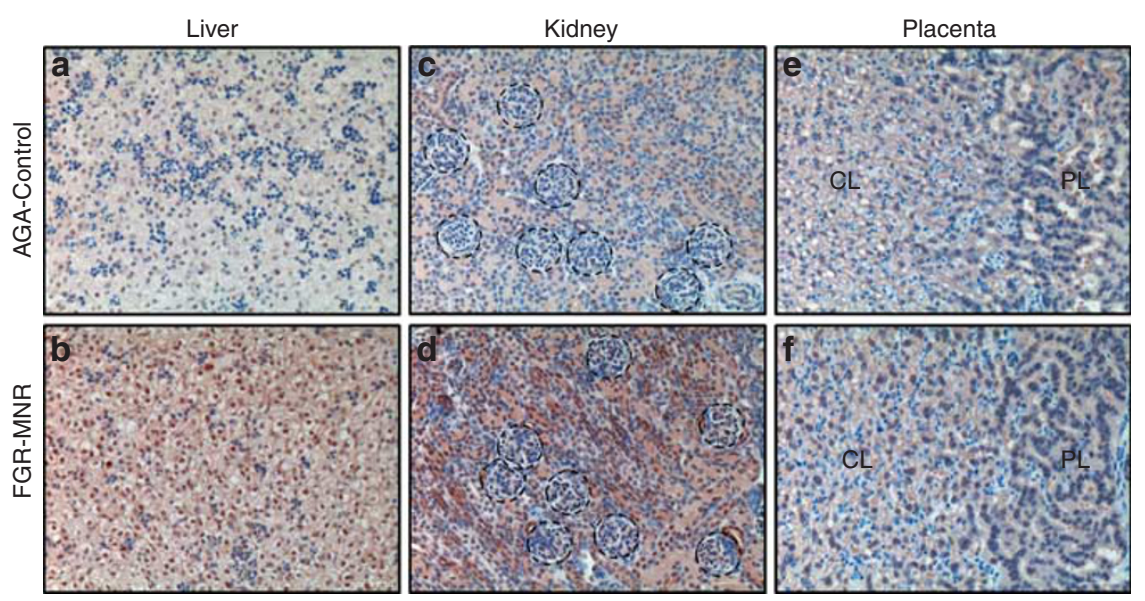

Figure 4. Representative photomicrographs illustrating positively stained cells for Hypoxyprobe-1 (brown) in the liver of an AGA-Control (a) and FGR-MNR (b) fetus showing the staining to be widely dispersed within the hepatocytes but increased in FGR-MNR fetuses; in the renal cortex of an AGA-Control (c) and FGR-MNR (d) fetus showing the staining to be less in the glomeruli (outlined) than in the surrounding proximal convoluted tubule and increased in FGR-MNR fetuses; and in the labyrinthine zone of the placenta of an AGA-Control (e) and FGR-MNR (f) fetus showing the staining to be more so peripherally (PL) than centrally $(\mathrm{CL})$ within the labyrinthine lobules, but with no difference between FGR-MNR and AGA-Control fetuses. Sections were also counterstained with hematoxylin. Bar $=50 \mu \mathrm{m}$. AGA, appropriate for gestational age; FGR, fetal growth restriction; MNR, maternal nutrient restriction.

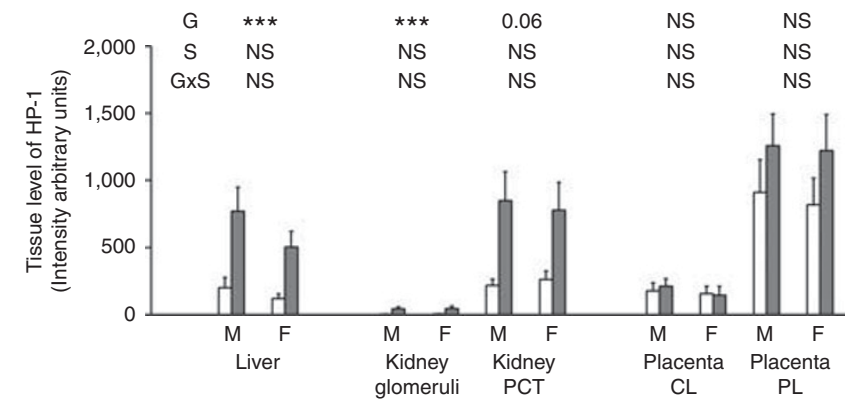

Figure 5. Hypoxyprobe-1 immunoreactivity levels in the fetal liver, renal cortex glomeruli, and surrounding proximal convoluted tubules (PCT), and centrally $(\mathrm{CL})$ and peripherally $(\mathrm{PL})$ within the placental labyrinthine lobules, for the male and female AGA-Control fetuses (open bars, $n=8$ and 8 ) and male and female FGR-MNR fetuses (gray bars, $n=8$ and 8). Data presented as mean staining intensity $\pm \mathrm{SEM} ;{ }^{* * *} P<0.001$. AGA, appropriate for gestational age; FGR, fetal growth restriction; $G$, group; MNR, maternal nutrient restriction; NS, not significant; $S$, sex..

although both males and females showed similar increases in their hemoglobin values (18). Mechanisms underlying these differences are not readily apparent, and Clemons et al. (28) found no sexual dimorphism in the EPO response of fetal and neonatal rats to induced hypoxia. However, there are known to be sex-related differences in responses to hypoxia, with females showing better adaptation than males, including the ventilatory and erythropoietic system responses that are tightly linked in females but not in males (29).

EPOR is a cell surface transmembrane protein to which EPO must bind in order to achieve signal transduction in both erythroid and non-erythroid tissues (19,30,31). As such, EPOR is found in both the liver and the bone marrow during fetal development in relation to the ontogeny for erythropoiesis $(31,32)$. However, EPO has also been reported to have nonhematologic functions, including the promotion of angiogenesis and cellular proliferation/differentiation, as well as cytoprotection with hypoxic conditions $(26,30)$. Accordingly, EPOR has been found in other fetal tissues, including the kidney and the placenta $(26,32)$. While the signaling for EPOR expression in tissues may variably involve HIF-1, nitric oxide, or even EPO itself, a primary stimulus for all of these will still be tissue hypoxia $(19,30)$. Similar to the EPO findings, EPOR levels were increased in female FGR-MNR liver tissues, although this was not statistically significant, consistent with some degree of local hypoxic stimulus and the adaptive need for increased erythropoiesis. However, there was no change in EPOR levels in the kidney or placenta in the female FGR-MNR fetuses despite the noted increase in EPO levels seen here, suggesting that nonhematologic EPO-EPOR responses do not occur with chronic hypoxemia, at least for the kidney and the placenta.

VEGF includes a family of glycoprotein hormones, with VEGF-A being the most abundant, which play a prominent role as regulators of vascular development and have been reported in several fetal tissues including the liver, kidney, and placenta $(33,34)$. Although the expression of VEGF is influenced by several factors, a major stimulus for the upregulation of VEGF is local tissue hypoxia mediated by HIF-1 (19,33). Similar to the EPO and EPOR findings, VEGF protein was increased in female FGR-MNR liver tissues, which is again consistent with some degree of local hypoxic stimulus. However, VEGF levels in the kidney and placenta of the female FGR-MNR fetuses remained unchanged, suggesting that the increased liver values might somehow relate to increased erythropoiesis here, rather than to an angiogenic stimulus. It is thus of note that in mice erythropoiesis in the fetal liver is impaired when hypoxic induction of VEGF is lacking, indicating a role for VEGF in erythropoiesis in the fetal liver (34). The unchanged VEGF levels in the liver and 
kidney of the male FGR-MNR fetuses likewise support a VEGF-erythropoiesis linkage rather than an angiogenic linkage. Here, the absence of change in the liver might be due to an earlier transition in erythropoeisis from the liver to the bone marrow in FGR males that might also explain the lower EPOR levels seen here.

In the present study, HP-1 staining was similarly seen in the male and female AGA-Control animals for each of the liver, kidney and placenta, indicating some level of local tissue hypoxia even under normal physiologic conditions in the fetus, which was also noted by Oh et al. (21) in near-term fetal guinea pigs. HP-1 staining in the liver was widely seen within the hepatocytes, while in the kidney this was considerably less in the glomeruli than in the surrounding proximal convoluted tubule area. This is consistent with renal blood flow initially to the glomeruli, and thereby better oxygenation, prior to passing through the surrounding cortical tissue as reported for the adult kidney (27). HP-1 was substantially increased in the liver and kidney cortical tissues of both male and female FGR-MNR fetuses, indicating lower levels of oxygenation in these tissues relative to those of the respective AGA-Control fetuses. HP-1 staining in the placenta was also widely seen, but more so peripherally and less so centrally within the labyrinthine lobules. This is consistent with the countercurrent blood flow described for the guinea pig placenta whereby maternal blood flows from arterial channels at the center of the lobule to venous channels in the interlobium, while fetal blood flows from fetal arteries in the interlobium to fetal veins at the center of the lobule, with the center lobule tissue thereby better oxygenated (23). Despite the substantial increases in HP-1 staining in the liver and kidney of FGRMNR fetuses, there was no evident change in the placental tissues of these animals. Since uteroplacental blood flow supplies oxygen to the placenta before it does to the fetus, placental tissue will normally be better oxygenated than fetal tissue (3). This may be further exaggerated under hypoxic conditions by nitric-oxide-mediated vasodilation within the placenta's central region as a mechanism to maintain oxygen homeostasis, albeit at the expense of fetal oxygenation (35). Of note, the extent of pimonidazole binding in hypoxic tissue will depend on tissue levels as well as the rate of bioreductive activation, and we therefore cannot exclude the possibility that group differences in placental transport of pimonidazole have impacted the HP-1 findings. However, any impact on placental transport is likely minimal since there were no differences in placental HP-1 IR between the two groups and this should not negate the conclusions drawn for the liver and kidney given the substantial changes in HP-1 IR seen here between the two groups.

Moderate MNR in guinea pigs beginning before pregnancy and continuing through pregnancy results in fetuses that have asymmetrical growth restriction and increased placental-fetal weight ratios as is often seen in human infants with growth restriction $(2,6,9,17,18)$. These FGR-MNR fetuses are also leaner, hypoglycemic, and polycythemic, which are again well associated with moderate growth restriction during human pregnancy $(4,5,17,18,36,37)$. These findings, along with the altered vascular development and structural changes reported in the placenta of guinea pigs subjected to moderate MNR, raise the likelihood of chronic hypoxemia as seen in human FGR pregnancies with suspected placental insufficiency $(5,6,12,36)$. The present findings of increased EPO and VEGF protein levels in the female FGR-MNR fetuses provide support for some degree of hypoxic stimulus in these fetal tissues and linkage to an adaptive need for increased erythropoiesis. The increased HP-1 staining in the liver and kidneys of the female FGR-MNR animals as an index of local tissue hypoxia provides further support for this conjecture. However, EPO and VEGF protein levels in the male FGR-MNR fetuses were not similarly increased, indicating sex-related differences in this response. This cannot be attributed to less hypoxic stimulus since HP-1 tissue staining was similarly increased from control levels in both male and female FGR-MNR fetuses and growth and metabolite characteristics did not differ (18). More likely is the better adaptation to hypoxia in females than in males, including the tighter link between oxygenation and increased erythropoiesis as previously noted (29). The present findings thereby provide further support for chronic hypoxia as a primary signaling mechanism for the decreased fetal growth with moderate MNR in guinea pig pregnancies. This may be a consequence of the altered vascularization and structural changes in the placentas of these animals $(12,13)$ and decreased placental blood flow as seen in rodent pregnancies with dietary restriction (38). However, the fetal hypoxia here appears to be largely postplacental in nature and likely also involves active enhancement of placental oxygenation at the expense of the fetus as noted (35). Importantly, chronic hypoxia is also likely to be a signaling mechanism for the programming of longer-term adverse outcomes in pregnancies with maternal undernutrition leading to FGR, which has added importance given the extensive use of MNR for the study of fetal programming $(1,12,13,17,18)$ and the role of maternal undernutrition as a primary causative factor for human FGR $(2,10,11)$.

\section{METHODS}

\section{Animal Cohorts and Tissue Collection}

An established model of moderate MNR in guinea pigs $(12,17)$ was used, with all experimental procedures approved by The University of Western Ontario Animal Use Subcommittee. Animal feeding, breeding, and pregnancy outcomes have previously been reported (18). Briefly, guinea pig sows were assigned to either a Control group fed ad libitum or an MNR group fed $70 \%$ of the average food intake per $\mathrm{kg}$ of body weight of the control animals from 4 weeks before conception until mid-pregnancy, increasing to $90 \%$ thereafter. On day $60-61$ of pregnancy (term $=\sim 68$ days), the hypoxia marker pimonidazole hydrochloride (Hypoxyprobe-1, $60 \mathrm{mg} / \mathrm{kg}$, Chemicon, Temecula, CA) was injected intraperitoneally into Control and MNR sows and allowed to circulate for $120 \mathrm{~min}$. Animals were then sedated, followed by laparotomy and delivery of each of the fetuses. Body and placental weights were obtained from all fetuses, along with crown rump length measurements. Fetuses were considered to be AGA if they weighed $\geq 80 \mathrm{~g}$ and FGR if $<80 \mathrm{~g}$, which is in accordance with the weight criteria we (39) and others (40) have used for categorizing AGA and FGR fetal weights in guinea pigs near 


\section{Articles Elias et al.}

term. Subsequently, only AGA fetuses from Control group litters and FGR fetuses from MNR group litters were subjected to full necropsy, which consisted of dissection and weighing of the brain, heart, and liver, extraction of the gonads for determining fetal sex, and extraction of the kidneys, skeletal muscle, and peri-renal adipose tissue. These organs/tissues along with the placenta were similarly partitioned in all animals and both frozen in liquid nitrogen and fixed in $4 \%$ paraformaldehyde for later analysis. Liver (right lobe), kidney, and placental tissues were assessed for hypoxia markers. Tissue for protein analysis was stored at $-80^{\circ} \mathrm{C}$ until protein quantification, and tissue for histology was kept in $4 \%$ paraformaldehyde for $72 \mathrm{~h}$, then washed in phosphate-buffered saline daily for 3 days and placed in 70\% ethanol for 7-14 days, prior to tissue processing and embedding in paraffin. Tissue sections were subsequently cut at $5 \mu \mathrm{m}$ thickness on a rotary microtome and mounted on Superfrost Plus slides (VWR Scientific, West Chester, PA).

Sixteen AGA-Control fetuses (8 male and 8 female) and 16 FGRMNR fetuses ( 8 male and 8 female) were selected for hypoxic tissue analysis. These animals were selected on the basis of no more than one male and one female from each litter; when two or more fetuses of the same sex met the weight criteria for study from the same litter, selection was made from the most medial position in the uterine horn.

\section{Protein Extraction and Western Blot Analysis}

Approximately $30-50 \mathrm{mg}$ of liver, kidney, or placental tissue was homogenized with $10 \mu \mathrm{l}$ of RIPA buffer per mg of kidney and placental tissue and $15 \mu \mathrm{l}$ of RIPA buffer per $\mathrm{mg}$ of liver tissue in the presence of a protease inhibitor (Roche, Mississauga, ON, Canada). Homogenates were sonicated and then centrifuged at $15,000 \mathrm{~g}$ for $10 \mathrm{~min}$ at $4{ }^{\circ} \mathrm{C}$, with $300 \mu \mathrm{l}$ of the supernatant extracted and retained as the total protein fraction. Equal amounts of tissue extracts were normalized by colorimetric BCA Protein Assay (Pierce, Madison, WI). Samples were stored at $-20^{\circ} \mathrm{C}$ until protein analysis.

The extracted protein samples were used to prepare loading samples for gel electrophoresis at a voltage of $180 \mathrm{~V}$ in gradient NuPAGE $4-12 \%$ Bis-Tris polyacrylamide gels (Invitrogen Life Technologies, Burlington, ON, Canada) for $1 \mathrm{~h}$. Male AGA-Control and FGR-MNR samples were loaded and run together on one gel, while female AGA-Control and FGR-MNR samples were run together on a separate gel. This was followed by gel transfer onto a polyvinyliden difluoride membrane (Millipore, Etobicoe, ON, Canada) at a voltage of $100 \mathrm{~V}$ for $2 \mathrm{~h}$. Following transfer, the membranes were thoroughly washed and then blocked for $2 \mathrm{~h}$ with $5 \%$ albumin in Tris-buffered saline containing $0.1 \%$ Tween. Membranes were then incubated overnight at $4{ }^{\circ} \mathrm{C}$ using primary antibodies specific for the protein targets, EPO (1:500, NBP1-84836, Novus Biologicals, Oakville ON, Canada), EPOR (1:500, H-194, sc5624, Santa Cruz Biotechnology, Dallas, TX), and VEGF-A (1:500 for liver and kidney; 1:300 for placenta, A-20, sc-152, Santa Cruz Biotechnology), diluted in 5\% non-fat milk $1 \times$ Tris-buffered salineTween 20 buffer. The following day, membranes were washed with phosphate-buffered saline and then probed for $1 \mathrm{~h}$ at room temperature with Horseradish peroxidase-conjugated donkey antirabbit IgG $(1: 10,000$, Jackson ImmunoResearch Laboratories, West Grove, PA), diluted in 5\% milk. Immunoreactive bands were visualized using a Luminata Forte Western HRP enhanced chemiluminesence detection system (Thermo Scientific, Nepean, ON, Canada) and VersaDoc Imaging System (BioRad Laboratories, Mississauga, ON, Canada). Densitometry analysis of protein bands was performed using ImageLab with signal saturation software (BioRad Laboratories) normalized to $\beta$-actin as a loading control. $\mathrm{EPO}, \mathrm{EPOR}$, and VEGF protein levels normalized to the protein levels of $\beta$-actin are presented as the mean change for FGR-MNR males from those of AGA-Control males and for FGR-MNR females from those of AGA-Control females.

\section{Hypoxyprobe-1 Immunohistochemistry}

Hypoxyprobe-1, a pimonidazole hydrochloride, is reduced by nitroreductases in relatively hypoxic cells $\left(\mathrm{pO}_{2}<10 \mathrm{~mm} \mathrm{Hg}\right)$ to form covalent protein adducts that can then be detected immunohistochemically using the Hypoxyprobe-1 kit (20-22). HP-1 IR was assessed by avidin-biotin-peroxidase immunohistochemistry (Vectastain Elite Kit; Vector Laboratories, Burlingame, CA). All slides were stained the same day using the same solutions to minimize variation in the intensity of the stain. Negative controls included substitution of the primary antiserum with nonimmune serum to rule out nonspecific binding, and confirmed absence of staining. All incubations were performed in a covered humidity chamber. Rinses between steps were performed in phosphate-buffered saline, and antibodies were diluted in Universal Antibody Diluent (Dako Canada, Burlington, ON, Canada).

Tissue sections were deparaffinized in xylene and rehydrated through graded ethanol baths. The slides were subjected to antigen target retrieval in $10 \mathrm{~mm}$ sodium citrate at $\mathrm{pH} 6.0$ for $20 \mathrm{~min}$ in a $90^{\circ}$ $\mathrm{C}$ vegetable steamer. Endogenous peroxidase was quenched in $1 \%$ hydrogen peroxide for $10 \mathrm{~min}$. Endogenous biotin was then blocked as directed using an avidin-biotin blocking kit (Vector Laboratories), and nonspecific antibody binding was blocked for 5 min using Background SNIPER (Biocare Medical, Concord, CA).

The slides were incubated overnight with rabbit polyclonal hypoxyprobe- 1 (Hypoxyprobe, Burlington, MA) at $4{ }^{\circ} \mathrm{C}$. They were then incubated for $40 \mathrm{~min}$ with biotinylated goat anti-rabbit IgG (Vector Laboratories), followed by $30 \mathrm{~min}$ with avidin-biotinperoxidase complex (Vector Laboratories), at room temperature. Bound antibody was developed with Cardassian 3,3-diaminobenzadine substrate (Biocare Medical) for $2 \mathrm{~min}$, with slides then counterstained for $30 \mathrm{~s}$ in Harris' Hematoxylin (Fisher Scientific, Ottawa, ON, Canada) at room temperature. The slides were then dehydrated through graded ethanol baths, cleared in xylene, mounted with Permount (Fisher Scientific, Toronto, ON, Canada), and dried.

HP-1 IR was imaged in four randomly selected high-power fields (HPF, $\times 20$ magnification) from comparable areas in the liver, renal cortex, and labyrinthine region of the placenta, which provided a stable running average for the tissue regions/sub-regions examined in each animal using a Zeiss Axiolmager Z1 microscope (Carl Zeiss Canada, Toronto, ON, Canada). Intensity of staining as a measure of tissue levels of pimonidazole (22) was determined using Image Pro Premier 7.0 software (Media Cybernetics, Rockville, MD). To ensure consistency and impartial evaluation, an automated macro incorporating a binary color intensity cutoff threshold was set up for each of the tissues examined based on advanced testing of a random sample of images, including screening against negative controls, to select and count only cell bodies deemed to be positively stained. While a different threshold and automation macro was thereby established for each of the three tissues, once set, the same macro was used for each image of that tissue. The scoring of images for a given tissue was completed within the same day and all analyses were performed with the investigator blinded to the animal cohort grouping. Intensity of staining was expressed as the mean intensity in arbitrary units for the 4 HPFs for each of the three tissues examined for each of the AGAControl and FGR-MNR fetuses.

\section{Data Acquisition and Statistical Analysis}

Fetal population characteristics, hypoxia-related protein levels, and HP-1 IR for the AGA-Control fetuses and FGR-MNR fetuses are presented as group means \pm SEM. Group findings were compared using analysis of variance to determine the effects of MNR and sex, except for the AGA-Control within-animal comparisons of kidney and placental sub-regional HP-1 IR, for which paired Student's $t$-test was used. Where interactions between MNR and sex were present or tending with $P<0.10$, post hoc tests were carried out to determine the effect of MNR in males and females separately (Graphpad Software, San Diego, CA). For all analyses, statistical significance was assumed at $P<0.05$. 


\section{Maternal undernutrition in guinea pigs $\quad$ Articles}

\section{ACKNOWLEDGMENTS}

We thank Dr Dan Hardy for his input into this study, Dr Lin Zhao for technical support with the protein analysis, Mr Larry Stitt for statistical support, and Ms Jennifer Ryder for technical assistance with the manuscript.

\section{STATEMENT OF FINANCIAL SUPPORT}

Supported by grants from the Children's Health Research Institute and Department of Obstetrics and Gynecology, University of Western Ontario, London, Canada (BSR, TRHR). BSR is the recipient of a Canada Research Chair Tier I in Fetal and Neonatal Health and Development.

\section{Disclosure: The authors declare no conflict of interest}

\section{REFERENCES}

1. Jansson T, Powell TL. Role of the placenta in fetal programming. underlying mechanisms and potential interventional approaches. Clin Sci (Lond) 2007;113:1-13.

2. Resnik R, Creasy RK. Intrauterine growth restriction. In: Creasy RK, Resnik R, Iams JD, et al., eds. Maternal-Fetal Medicine. Philadelphia, PA: Elsevier Sauders, 2014.

3. Kingdom JC, Kaufmann P. Oxygen and placental villous development: origins of fetal hypoxia. Placenta 1997;18:613-21.

4. Ferrazzi E, Rigano S, Bozzo M, et al. Umbilical vein blood flow in growthrestricted fetuses. Ultrasound Obstet Gynecol 2000;16:432-38.

5. Soothill PW, Nicolaides KH, Campbell S. Prenatal asphyxia, hyperlacticaemia, hypoglycaemia, and erythroblastosis in growth retarded fetusesBr Med J (Clin Res Ed) 1987;294:1051-53.

6. Lackman F, Capewell V, Gagnon R, Richardson B. Fetal umbilical cord oxygen values and birth to placental weight ratio in relation to size at birth. Am J Obstet Gynecol 2001;185:674-82.

7. Jansson T, Thordstein M, Kjellmer I. Placental blood flow and fetal weight following uterine artery ligation. Temporal aspects of intrauterine growth retardation in the guinea pig. Biol Neonate 1986;49:172-80.

8. Murotsuki J, Challis JR, Han VK, Fraher LJ, Gagnon R. Chronic fetal placental embolization and hypoxemia cause hypertension and myocardial hypertrophy in fetal sheep. Am J Physiol 1997;272:R201-7.

9. Lumey LH. Compensatory placental growth after restricted maternal nutrition in early pregnancy. Placenta 1998;19:105-1.

10. Sebire NJ, Jolly M, Harris J, Regan L, Robinson S. Is maternal underweight really a risk factor for adverse pregnancy outcome? A population-based study in London. BJOG 2001;108:61-6.

11. Ehrenberg HM, Dierker L, Milluzzi C, Mercer BM. Low maternal weight, failure to thrive in pregnancy, and adverse pregnancy outcomes. Am J Obstet Gynecol 2003;189:1726-30.

12. Roberts CT, Sohlstrom A, Kind KL, et al. Maternal food restriction reduces the exchange surface area and increases the barrier thickness of the placenta in the guinea-pig. Placenta 2001;22:177-85.

13. Belkacemi L, Nelson DM, Desai M, Ross MG. Maternal undernutrition influences placental-fetal development. Biol Reprod 2010;83:325-1.

14. Aherne W, Dunnill MS. Morphometry of the human placenta. Br Med Bull 1966;22:5-8.

15. Gaccioli F, Lager S, Powell TL, Jansson T. Placental transport in response to altered maternal nutrition. J Dev Orig Health Dis 2013;4:101-5.

16. Carter AM. Animal models of human placentation-a review. Placenta 2007;28 (Suppl A): S41-7.

17. Kind KL, Roberts CT, Sohlstrom AI, et al. Chronic maternal feed restriction impairs growth but increases adiposity of the fetal guinea pig. Am J Physiol Regul Integr Comp Physiol 2005;288:R119-26.

18. Elias AA, Ghaly A, Matushewski B, Regnault TR, Richardson BS. Maternal nutrient restriction in guinea pigs as an animal model for inducing fetal growth restriction. Reprod Sci 2016;23:219-7.

19. Semenza GL. Regulation of oxygen homeostasis by hypoxia-inducible factor 1. Physiology (Bethesda) 2009;24:97-106.
20. Raleigh JA, Chou SC, Arteel GE, Horsman MR. Comparisons among pimonidazole binding, oxygen electrode measurements, and radiation response in C3H mouse tumors. Radiat Res 1999;151:580-9.

21. Oh C, Dong Y, Harman C, Mighty HE, Kopelman J, Thompson LP. Chronic hypoxia differentially increases glutathione content and gammaglutamyl cysteine synthetase expression in fetal guinea pig organs. Early Hum Dev 2008;84:121-7.

22. Rueda-Clausen CF, Morton JS, Lopaschuk GD, Davidge ST. Long-term effects of intrauterine growth restriction on cardiac metabolism and susceptibility to ischaemia/reperfusion. Cardiovasc Res 2011;90:285-94.

23. Miglino MA, Carter AM, Ambrosio CE, et al. Vascular organization of the hystricomorph placenta: a comparative study in the agouti, capybara, guinea pig, paca and rock cavy. Placenta 2004;25:438-8.

24. Eckardt KU, Kurtz A. Regulation of erythropoietin production. Eur J Clin Invest 2005;35 (Suppl 3): 13-9.

25. Teramo KA, Widness JA. Increased fetal plasma and amniotic fluid erythropoietin concentrations: markers of intrauterine hypoxia. Neonatology 2009;95:105-6.

26. Kowalska-Kanka A, Maciejewski T, Niemiec KT. The role and regulation of secretion of erythropoietin in pregnancy. Med Wieku Rozwoj 2013;17: 270-5.

27. Suzuki N. Erythropoietin gene expression: developmental-stage specificity, cell-type specificity, and hypoxia inducibility. Tohoku J Exp Med 2015;235:233-40.

28. Clemons GK, Fitzsimmons SL, DeManincor D. Immunoreactive erythropoietin concentrations in fetal and neonatal rats and the effects of hypoxia. Blood 1986;68:892-9.

29. Soliz J, Khemiri H, Caravagna C, Seaborn T. Erythropoietin and the sexdimorphic chemoreflex pathway. Adv Exp Med Biol 2012;758:55-62.

30. Beleslin-Cokic BB, Cokic VP, Yu X, Weksler BB, Schechter AN, Noguchi CT. Erythropoietin and hypoxia stimulate erythropoietin receptor and nitric oxide production by endothelial cells. Blood 2004;104:2073-80.

31. David RB, Sjaastad OV, Blom AK, Skogtvedt S, Harbitz I. Ontogeny of erythropoietin receptor mRNA expression in various tissues of the foetal and the neonatal pig. Domest Anim Endocrinol 2005;29:556-63.

32. David RB, Lim GB, Moritz KM, Koukoulas I, Wintour EM. Quantitation of the mRNA levels of Epo and EpoR in various tissues in the ovine fetus. Mol Cell Endocrinol 2002;188:207-18.

33. Gourvas V, Dalpa E, Konstantinidou A, Vrachnis N, Spandidos DA, Sifakis S. Angiogenic factors in placentas from pregnancies complicated by fetal growth restriction (review). Mol Med Rep 2012;6:23-7.

34. Rehn M, Kertesz Z, Cammenga J. Hypoxic induction of vascular endothelial growth factor regulates erythropoiesis but not hematopoietic stem cell function in the fetal liver. Exp Hematol 2014;42: 941-4.

35. Kulandavelu S, Whiteley KJ, Bainbridge SA, Qu D, Adamson SL. Endothelial NO synthase augments fetoplacental blood flow, placental vascularization, and fetal growth in mice. Hypertension 2013;61:259-66.

36. Economides DL, Nicolaides KH. Blood glucose and oxygen tension levels in small-for-gestational-age fetuses. Am J Obstet Gynecol 1989;160: 385-9.

37. Kramer MS, Olivier M, McLean FH, et al. Impact of intrauterine growth retardation and body proportionality on fetal and neonatal outcome. Pediatrics 1990;86:707-13.

38. Ahokas RA, Anderson GD, Lipshitz J. Cardiac output and uteroplacental blood flow in diet-restricted and diet-repleted pregnant rats. Am J Obstet Gynecol 1983;146:6-13.

39. Piorkowska K, Thompson J, Nygard K, Matushewski B, Hammond R, Richardson B. Synaptic development and neuronal myelination are altered with growth restriction in fetal guinea pigs. Dev Neurosci 2014;36: 465-76.

40. Jansson T, Persson E. Placental transfer of glucose and amino acids in intrauterine growth retardation: studies with substrate analogs in the awake guinea pig. Pediatr Res 1990;28:203-8. 